\title{
Current Surgical Attitude Towards Hydatid Liver Disease - Experience of Ist Surgical Department of the Emergency Hospital of Craiova
}

\author{
Cirju B1 ${ }^{1}$, Patrascu St ${ }^{1}$, Margaritescu D², Nemes R², Georgescu I² \\ $1^{\text {st }}$ Clinic of Surgery, Emergency County Hospital, Craiova, Romania \\ 2 Department of Surgery, University of Medicine and Pharmacy, Craiova, Romania
}

\begin{abstract}
Introduction: The aim of this study is the evaluation of the diagnostic and therapeutic strategy in liver hydatid disease.
Methods: We analyzed 97 cases of liver hydatid cyst (63 females/34 males, aged between 5 and 85 years) hospitalized in the Ist Surgical Department of the Emergency County Hospital of Craiova between 2000 and 2012. The diagnosis was established clinically, by laboratory and imaging tests.

Results: The hydatid cysts were unilocular in 60 cases, the majority (68.31\%) being located on the right liver, multilocular in 39 cases, located on both lobes of the liver (9.9\%); 17 patients had other locations of the cysts as well (spleen 5 cases, peritoneum 12 cases). Fourty-five (46.39\%) cases were uncomplicated and the other 52 cases presented one ore more evolutionary complications: biliary - 44 (43.36\%), infection - 15 (14.85\%), rupture into peritoneum - 12 cases and bilio-bronchial fistula in 1 case. All patients were operated: 90 patients by open surgery and 7 by laparoscopic approach. Albendazole was administered as prophylaxis of postoperative recurrence. In 73 cases (72.27\%) the evolution was favorable. We recorded 26 cases (25.74\%) of postoperative complications: infectious - 9 cases, external biliary fistula - 12 cases and general complications in 6 cases. The overall postoperative mortality was $2.06 \%$. We did not register any postoperative complication in the laparoscopic group.

Conclusions: Laparoscopic approach is both safe and feasible, with well codified indications. The most important factor to achieve a successful laparoscopic procedure is the adequate selection of patients. Both intraoperative and postoperative data clearly indicated that the minimal invasive approach provided superior results to open access surgery in terms of complications rate, early recovery and hospital stay.
\end{abstract}

Keywords: hepatic hydatid cyst, Echinococcus granulosus, laparoscopy, surgical procedures

Received: 17 April 2012 / Accepted: 25 February 2014

\section{Introduction}

Hydatid disease is an endemic parasitic condition caused by the larval forms of Echinococcus granulosus and Echinococcus multinodularis. Worldwide, hydatid disease currently remains one major public health problem, especially in rural areas $[1,2]$. Because of the disappointing results of medical treatment - which currently remains restricted to inoperable cases or in preventing recurrences, surgery is the main solution for cystic echinococcosis. Due to the technical challenges, the best surgical access and technique still remains controversial, laparoscopy offering, in specific conditions, an alternative to the more conventional open approach.

The aim of this retrospective study was to investigate the diagnostic tools and the feasibility of different treatment options in patients suffering of cystic hydatidosis.

\section{Patients and methods}

In this retrospective analysis we reviewed the medical records of all patients with hydatid disease who had been admitted in our institution from 2000 to 2012. Ninety-seven patients ( 63 females $/ 34$ males), mainly coming from rural areas $(71.13 \%)$, aged between 5 and 85 years old (maximal

Correspondence to: Theodora Benedek

E-mail: hintea_teodora@yahoo.com incidence $-43.29 \%$ in the $4^{\text {th }}$ to $6^{\text {th }}$ decades of life) were analyzed.

Criteria for inclusion of patients in the study comprised all cases previously diagnosed with liver-localized hydatid cyst with no adequate response to medical treatment, and patients having been incidentally diagnosed with hepatic hydatidosis, undergoing open or surgery laparoscopic surgery combined with traditional medical treatment.

Exclusion criteria for patients in the study covered all patients diagnosed with localized extrahepatic hydatid cyst, patients diagnosed with hepatic hydatid cyst smaller than $5 \mathrm{~cm}$ in diameter, undergoing medical treatment, patients diagnosed with calcified small size liver hydatid cyst which did not receive any treatment.

Necessary study data were extracted from patients' clinical observation sheets and surgical protocols and then were statistically analyzed. All patients evaluated in this study had the following parameters taken into account: demographic data (age, sex, living environment, and dynamics of admissions) that were statistically processed; clinical and paraclinical results to assess the relevance of various symptoms or diagnostic laboratory explorations, the results of different types of treatments, expressed through morbidity, number of reinterventions, mortality, length of stay, disease recurrence. 
All data collected for this study were stored in Windows Excel files (Microsoft Corp., Redmond, WA, USA). For statistical processing of data were used the following types of statistical tests: tests to measure the dependence between parameters - Pearson correlation coefficient, chi squared - as well as significance tests, specifically Anova and Student's t-tests, using SPSS 16.0 (SPSS Inc., Chicago, IL, USA).

\section{Results}

Preoperative diagnosis involved anamnesis, clinical examinations, laboratory and imaging tests (ultrasonography and computer tomography). Unilocular cysts were found in the majority of cases, usually located on the right liver, while multilocular cysts were identified in 39 cases, 10 located on both the right and left liver. Additional locations comprised the spleen and the peritoneum. The reported series consisted in 45 uncomplicated cases and 52 cases showing one ore more evolutionary complications. Biliary lesions accounted for the majority of complications (43.36\%), followed by infection and leakage into the peritoneum $(27.6 \%)$ We also registered one case of bilio-bronchical fistula (Table I).

The surgical treatment included open surgery (92 cases) and laparoscopy (7 cases). The type of access in the open subgroup was midline incision in 64 cases $(63.36 \%)$ and right subcostal (Kocher) incision in 28 cases $(27.72 \%)$. Regarding to the actual treatment of the cyst the conservative surgical techniques were the method of choice (68.31\%): pericystectomy with drainage of the residual cavity was applied in 62 patients $(61.38 \%)$, followed by omentoplasty in 4 cases $(3.96 \%)$. In three cases internal drainage was performed - cystogastrostomy or cystojejunostomy. Cyst resection was performed in 11 cases $(10.89 \%)$, atypical hepatectomy in 11 cases $(10.89 \%)$ and right hepatectomy in one case. The associated procedures included colecistectomy -50 patients $(49.5 \%)$, T-tube drainage -15 cases (14.85\%), choledocoduodenostomy -5 patients $(5.55 \%)$ and papilosfincterotomy -2 cases (1.98\%).

Laparoscopic surgery was performed in 7 cases - pericystectomy with drainage of the residual cavity - associ-

Table I. Type and distribution of echinococcal cysts

\begin{tabular}{lcc}
\hline Location and type of hepatic hydatid cyst & No of cases & $\%$ \\
\hline Primary & 89 & $88.11 \%$ \\
Recurrent & 10 & $9.9 \%$ \\
Uncomplicated & 47 & $46.53 \%$ \\
Complicated & 52 & $51.48 \%$ \\
Single & 60 & $59.4 \%$ \\
Multiple & 39 & $38.61 \%$ \\
Right + left liver lobe & 10 & $9.9 \%$ \\
Right liver lobe & 69 & $68.31 \%$ \\
Left liver lobe & 20 & $19.8 \%$ \\
Extrahepatic locations & 17 & $16.83 \%$ \\
$\quad$ Splenic & 5 & $4.95 \%$ \\
Peritoneal & 12 & $11.88 \%$ \\
\hline
\end{tabular}

ated to colecistectomy in 3 cases. The main indication for laparoscopy was small size, uncomplicated and peripheral cysts.

The postoperative therapy with Albendazole as prophylaxis for recurrences was instituted in all patients and was not associated with any side effects

Most of the cases $(72.27 \%)$ had a fair evolution. Five biliary fistulas and one abscess of the residual cavity required re-laparotomy. The hospitalization time varied widely (5-98 days). We registered 26 postoperative complications (Table II).

The most common complications were external biliary fistulae (12 cases); we also noticed one bilio-pleural fistula. Nine cases $(8.91 \%)$ presented infectious complications: subphrenic abscess ( 5 cases), infection of the residual cavity ( 3 cases) and supramesocolic peritonitis following a gastrocolic fistula (1 case). Excepting minor local bleeding, no intraoperative complications occurred during the laparoscopic procedures. Postoperative morbidity and mortality were null in the laparoscopic group. We registered 2 deaths with a postoperative mortality rate of $1.98 \%$, the cause of death being myocardial infarction and pulmonary embolism (1 case each).

We did not register any recurrence during the 13 years follow-up period.

\section{Discussions}

Although considered an uncommon presence in many Western countries but with a high incidence in the Mediterannean and Balkan region, the hydatid disease is the source of considerable socioeconomic problem in the rural areas of Romania too [2]. This is the reason why the treatment should immediately be instituted whenever the disease is diagnosed.

Surgery remained the method of choice in the treatment of cystic echinococcosis [3-5]. The main principles of hydatid cyst surgery, originally established more than half a century ago, included complete removal of the parasite and the treatment of the restant cavity, alongside with the preservation of healthy tissue and prevention of intraoperative

Table II. General postoperative morbidity after hydatid cyst surgery

\begin{tabular}{lcc}
\hline Morbidity & 26 & $25.74 \%$ \\
\hline Suppurative complications: & 9 & $8.91 \%$ \\
Subphrenic abscess & 5 & $4.95 \%$ \\
Infection of the residual cavity & 3 & $2.97 \%$ \\
Supramesocolic peritonitis due to & 1 & $0.99 \%$ \\
gastro-colic fistula & & \\
Biliary complications & 13 & $12.87 \%$ \\
External bile leaks & 12 & $11.88 \%$ \\
Postoperative bilio-pleural fistula & 1 & $0.99 \%$ \\
Hemorrhages & 1 & $0.99 \%$ \\
General complications & 6 & $5.94 \%$ \\
Myocardial stroke & 1 & $0.99 \%$ \\
Pleurisy & 4 & $3.96 \%$ \\
Alcoholic coma & 1 & $0.99 \%$ \\
\hline
\end{tabular}


spillage [6]. These principles can be achieved by either open surgery or by laparoscopy, the laparoscopic approach being increasingly used lately. Minimal invasive solutions in case of hydatid disease, including laparoscopy or USguided percutaneous aspiration, seem to be beneficial for the early recovery of the patient, considering that surgery for hydatid disease is usually followed by high postoperative morbidity, ranging from $4 \%$ to $40 \%[1,7-10]$. The laparoscopic tehnique offers an advantage of better visual control of the cyst cavity under magnification, which allows better detection of small open bile ducts that leak during the postoperative recovery period. The small number of cases involving the laparoscopic approach is a direct consequence of the recent introduction of this type of access for the cystic echinococcosis. Although we did not experience the percutaneous ultrasonographic solutions, the overall results of laparoscopy in our study provide a strong recommendation for the introduction of the minimal invasive therapies in the treatment of hydatid disease in well selected cases. The criteria of selection should exclude patients with a deep seated cyst, multiple cysts, and possible communication between the cyst and biliary tract should be excluded from laparoscopic approach.

Unfortunately extensive large scale randomised studies investigating intraoperative and postoperative complications for liver hydatidosis, especially concerning minimalinvasive surgery. Ramachandran et al. reported a number of 6 cases of hydatid cyst dealt by laparoscopic aproach, with no complication mentioned. Manterolla et al. reported only one case of biliary fistula out of 9 cases of hydatid cyst of the liver. Saglam et al, out of 11 cases of liver hydatid cyst treated by laparoscopic approach, performed only one conversion due to an intraoperative bleeding [16-18].

In our study the overall postoperative morbidity was in these limits (25.74\%) for open surgery and considerably lower for the laparoscopic approach, confirming other recent studies that investigated the impact of minimal access techniques. Secchi et al. recorded in a 2010 study a $26 \%$ rate of postoperative complications after radical surgical procedures compared with $45 \%$ complications after conservative surgical procedures [16]. However, our study clearly indicates that the perioperative results of laparoscopy can be further improved, especially on well selected cases.

Recurrence is usually observed after the spillage of live parasites into the peritoneal cavity and is rarely observed after the complete removal of an intact cyst [11]. In our study, although regular follow-up was performed, there was no registered postoperative recurrence, possibly in relation to the type of surgical technique (complete cyst removal) and to the postoperative drug therapy with Albendazole.

The selection of cases, as well as the relatively small number of cases in the laparoscopic group could be con- sidered as the major drawbacks of our study. Still, given the widespread use of laparoscopy in our department, the greater addressability and better diagnostic tools, the balance between the two types of surgical approaches will improve, thus offering more univocal results with stronger statistical impact.

\section{Conclusions}

Laparoscopic approach is both safe and feasible, with well codified indications. The most important factor to achieve a successful laparoscopic procedure is the adequate selection of patients. Both intraoperative and postoperative data clearly indicated that the minimal invasive approach provided superior results to open access surgery in terms of complications rate, early recovery and hospital stay.

\section{References}

1. Cirenei A, Bertoldi I. Evolution of surgery for liver hydatidosis from 1950 to today: analysis of a personal experience. World J Surg. 2001;25:87-92.

2. Motas C, Motas M, Davidescu M, Rus O, Horvath T. Hydatid cyst of anterior mediastinum. Chirurgia. 2012;107:115-118.

3. Alfieri S, Doglietto GB, Pacelli F, et al. Radical surgery for liver hydatid disease: a study of 89 consecutive patients. Hepatogastroenterology. 1997;44:496.

4. Kumar A, Lal BK, Chattopadhyay TK. Hydatid disease of the liver; surgical options. Trop. Gastroenterol. 1992;13:102.

5. Mentes A. Hydatid liver disease: a perspective in treatment. Dig. Dis. 1994;12:150.

6. Buttenschoen K, Carli Buttenschoen D. Echinococcus infection: the challenge of surgical treatment. Langenbecks Arch Surg. 2003;388:218230.

7. Saftoiu A, Gheonea DI, Georgescu I. Rezolvarea fistulelor biliare externe prin colangiografie endoscopică retrogradă, cu sfincterotomie și/sau stentare. Chirurgia. 2006;101(3):281-288.

8. Fisichella PM, Donaldson K, Helton WS. Hepatic and splenic hydatidosis managed with percutaneous aspiration, injection, and reaspiration (PAIR) of the hepatic cyst and laparoscopic splenectomy. J Gastrointest Surg. 2008;12:1615-1617.

9. Men S, Hekimoglu B, Yucesoy C, Arda IS, Baray I. Percutaneous treatment of hepatic hydatid cysts: an alternative to surgery. Am. J. Roentgenol. 1999;172:83-89.

10. Guibert L, Gayral F. Laparoscopic treatment of hepatic hydatid cyst. Surg. Laparosc. Endosc. 1998;8:280-282.

11. Sielaff TD, Taylor B, Langer B. Recurrence of the hydatid disease. World J Surg. 2011;25:83-86.

12. da Silva AM. Human Echinococcosis: A Neglected Disease. Gastroenterology Research and Practice Volume, 2010, Article ID 583297

13. Akcan A, Sozuer E, Akyildiz H, Ozturk A, Atalay A, Yilmaz Z. Predisposing factors and surgical outcome of complicated liver hydatid cysts. World $J$ Gastroenterol. 2010;16:3040-3048.

14. Foster EN, Hertz G. Echinococcus of the liver treated with laparoscopic hepatectomy. Perm J. 2010;14:45-46.

15. Misra MC, Khan RN, Bansal VK, et al. Laparoscopic pericystectomy for hydatid cyst of the liver. Surg Laparosc Endosc Percutan Tech. 2010;20: 24-26.

16. Secchi MA, Pettinari R, Mercapide C, et al. Surgical management of liver hydatidosis: a multicentre series of 1412 patients. Liver Int. 2010;30:8593.

17. Ramachandran CS, Goel D, Arora V. Laparoscopic surgery in hepatic hydatid cysts: a technical improvement. Surg Laparosc Endosc Percutan Tech. 2001;11:14-8

18. Manterola C, Fernandez O, Munoz S, et al. Laparoscopic pericystectomy for liver hydatid disease: description of results observed during a long follow-up period. Surg Endosc. 2002;16:521-4. 